\title{
CORRECTION
}

\section{Correction to: A Time-Domain Formulation of Elastic Waves in Heterogeneous Unbounded Domains}

\section{Boyoung Kim ${ }^{1}$. Jun Won Kang ${ }^{1}$}

Published online: 9 October 2019

๑) Korean Multi-Scale Mechanics (KMSM) 2019

\section{Correction to: Multiscale Science and Engineering (2019) 1:220-235 \\ https://doi.org/10.1007/s42493-019-00019-z}

There are a few errors in the published version of the article. Three equations should be corrected, and one equation should be added to the original article.

1. Eq. 22(b) on page 224 should appear as

$\mathcal{D}:[a \dddot{\mathbf{S}}+b \ddot{\mathbf{S}}+c \dot{\mathbf{S}}+d \mathbf{S}]$

$=\frac{1}{2}\left[(\nabla \ddot{\mathbf{u}}) \tilde{\boldsymbol{\Lambda}}_{e}+\tilde{\boldsymbol{\Lambda}}_{e}(\nabla \ddot{\mathbf{u}})^{\mathrm{T}}+(\nabla \dot{\mathbf{u}}) \tilde{\boldsymbol{\Lambda}}_{p}+\tilde{\boldsymbol{\Lambda}}_{p}(\nabla \dot{\mathbf{u}})^{\mathrm{T}}\right.$

$\left.+(\nabla \mathbf{u}) \tilde{\boldsymbol{\Lambda}}_{w}+\tilde{\boldsymbol{\Lambda}}_{w}(\nabla \mathbf{u})^{\mathrm{T}}\right]$,

2. Eq. 23(b) on page 224 should be corrected to

$\left(\ddot{\mathbf{S}}^{\mathrm{T}} \tilde{\boldsymbol{\Lambda}}_{e}+\dot{\mathbf{S}}^{\mathrm{T}} \tilde{\boldsymbol{\Lambda}}_{p}+\mathbf{S}^{\mathrm{T}} \tilde{\boldsymbol{\Lambda}}_{w}\right) \mathbf{n}=\mathbf{0} \quad$ on $\Gamma_{N}^{\mathrm{PML}} \times \mathbf{J}$,

3. Eq. 24(a) on page 224 should appear as

$$
\begin{aligned}
& \int_{\Omega} \nabla \mathbf{w}:\left(\ddot{\mathbf{S}}^{\mathrm{T}} \tilde{\boldsymbol{\Lambda}}_{e}+\dot{\mathbf{S}}^{\mathrm{T}} \tilde{\boldsymbol{\Lambda}}_{p}+\mathbf{S}^{\mathrm{T}} \tilde{\boldsymbol{\Lambda}}_{w}\right) d \Omega \\
& +\int_{\Omega} \mathbf{w} \cdot \rho(a \ddot{\mathbf{u}}+b \ddot{\mathbf{u}}+c \dot{\mathbf{u}}+d \mathbf{u}) d \Omega \\
& =\int_{\Gamma_{N}} \mathbf{w} \cdot\left(\ddot{\mathbf{S}}^{\mathrm{T}} \tilde{\boldsymbol{\Lambda}}_{e}+\dot{\mathbf{S}}^{\mathrm{T}} \tilde{\boldsymbol{\Lambda}}_{p}+\mathbf{S}^{\mathrm{T}} \tilde{\boldsymbol{\Lambda}}_{w}\right) \mathbf{n} d \Gamma+\int_{\Gamma_{N}} \mathbf{w} \cdot a \dot{\mathbf{f}} d \Omega .
\end{aligned}
$$

4. The following equation should be added on page 225 of the original article.

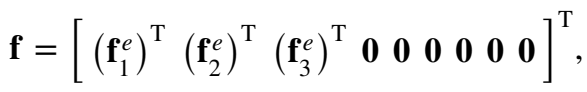

None of the corrections affect the conclusions of the article.

The original article can be found online at https://doi.org/10.1007/ s42493-019-00019-z.

Jun Won Kang

jwkang@hongik.ac.kr

Boyoung Kim

by900824@daum.net

1 Department of Civil Engineering, Hongik University, Seoul 04066, Korea 